\title{
QuickAutoML: Uma ferramenta para treinamento automatizado de modelos de aprendizado de máquina
}

\author{
Guilherme Siqueira $^{1}$, Gustavo Rodrigues ${ }^{1}$, Eduardo Feitosa ${ }^{2}$, Diego Kreutz ${ }^{1}$ \\ ${ }^{1}$ Universidade Federal do Pampa (Unipampa) \\ ${ }^{2}$ Universidade Federal do Amazonas (UFAM) \\ \{NomeSobrenome\}.aluno@unipampa.edu.br \\ efeitosalicomp.ufam.br,kreutz@unipampa.edu.br
}

\begin{abstract}
Resumo. Com o aumento da popularidade dos modelos de aprendizado de máquina em diferentes domínios e contextos, aumentou também a necessidade por ferramentas e mecanismos capazes de facilitar a otimização e utilização prática desses modelos. Neste trabalho, apresentamos a QuickAutoML, uma ferramenta que automatiza os processos de criação, treinamento, ajuste de hiper-parâmetros e validação de modelos. O principal objetivo da ferramenta é abstrair a complexidade desses processos para permitir que usuários com pouco conhecimento técnico possam criar modelos robustos em poucos minutos.
\end{abstract}

\section{Introdução}

Aprendizado de máquina (ou machine learning) tem como objetivo melhorar o desempenho de uma solução computacional motivado por determinado problema (e.g., classificar malwares) através de aprendizado contínuo [Yao and Liu, 2014]). O desenvolvimento de soluções baseadas em aprendizado de máquina envolve diversos processos como tratamento e manipulação de dados, engenharia de características (ou feature engineering) e seleção de algoritmos. O fato de tais processos muitas vezes ocorrerem de forma ad hoc, aliado à necessidade de conhecimento do domínio do problema a ser solucionado, torna o desenvolvimento de modelos de aprendizado de máquina custoso em tempo, dependente de conhecimento técnico especializado e difícil de ter sua viabilidade avaliada.

Ferramentas de automação de processos de aprendizado de máquina, como TPOT [Olson and Moore, 2016] e auto-sklearn [Feurer et al., 2015], permitem obter artefatos testáveis com um custo de tempo menor em comparação com abordagens manuais. Em particular, essas ferramentas reduzem substancialmente a curva de aprendizagem uma vez que eliminam a necessidade de conhecimentos específicos da área de aprendizado de máquina. Entretanto, o usuário ainda necessita realizar ajustes finos manualmente (e.g., definição do espaço de busca, configurações de CPU e memória), o que elimina apenas parcialmente o problema da dependência de conhecimento especializado. Além disso, essas ferramentas consomem tipicamente horas (ou até mesmo dias) para explorar as opções de otimização, seleção e treinamento de modelos, aumentando o custo computacional da validação inicial de um projeto de aprendizado de máquina.

Neste trabalho, propomos a QuickAutoML, que permite desenvolver modelos de aprendizado de máquina sem necessidade de conhecimento técnico especializados. A ferramenta identifica o algoritmo que melhor se ajusta ao problema e realiza sua otimização através de ajustes finos automatizados (i.e., seleciona automaticamente hiperparâmetros 
otimizados). Em uma análise experimental (Seção 3), demonstramos, utilizando seis datasets distintos, que a QuickAutoML consegue atingir resultados semelhantes a ferramentas TPOT, em termos de acurácia, porém com um tempo de execução consideravelmente menor.

\section{Implementação}

A QuickAutoML é um arcabouço para os algoritmos fornecidos pela scikit-learn, uma biblioteca que implementa diversos algoritmos de aprendizado de máquina para a linguagem Python [Pedregosa et al., 2011]. A partir dos algoritmos, o processo de treinamento de modelos é ilustrado na Figura 1.

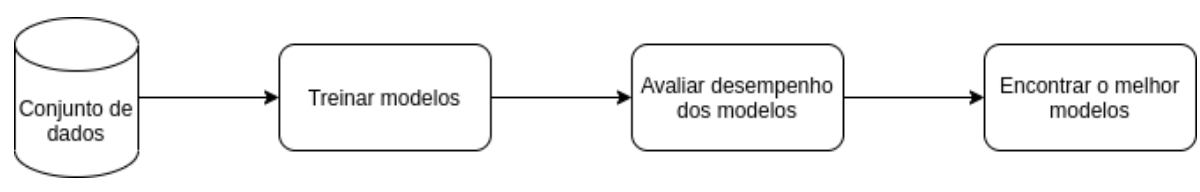

Figura 1. Processo de treinamento

O ponto de partida é o conjunto de dados (i.e., dataset) que será utilizado para treinamento, separado em dados de entrada e dados de saída, de forma que cada amostra de entrada (variável preditora) esteja associada a uma amostra de saída (variável alvo). A próxima etapa é treinar os modelos a fim de identificar o que melhor se ajusta aos dados; o parâmetro são as métricas de avaliação. Nesse treinamento, são criados e testados diversos candidatos com diferentes configurações.

\subsection{Métricas de avaliação}

Métricas de avaliação podem ser definidas como aspectos de desempenho de um modelo, tornando mensurável a diferença entre o real e o que foi predito [Botchkarev, 2018]. Para realizar a comparação entre diferentes modelos, é essencial definir uma métrica que servirá como parâmetro para a avaliação. A escolha entre diferentes métricas pode influenciar diretamente no resultado da comparação, pois diferentes modelos tendem a apresentar valores diferentes para um conjunto de métricas [Caruana and Niculescu-Mizil, 2006].

A escolha da métrica reflete diretamente os requisitos do problema. Por exemplo, em aplicações onde é mais crítico reduzir a taxa de falsos negativos gerados na classificação, uma alta revocação é mais significante para determinar a qualidade do modelo do que uma alta precisão. Por padrão, a métrica utilizada para comparação dos modelos é a acurácia (i.e., a porcentagem de predições corretas feitas pelo modelo), porém, o usuário pode utilizar outras métricas de classificação (precisão, revocação ou F1 score).

\subsection{Treinamento e seleção de modelos}

O processo de treinamento é iniciado através da definição de uma lista de potenciais candidatos a modelos. Essa lista é populada em duas etapas: primeiramente, definimos as famílias de algoritmos, como métodos baseados em bagging ou em distância. Para cada algoritmo é fornecida uma combinação diferente de hiperparâmetros (i.e., valores que controlam o processo de aprendizado de determinado modelo [Wu et al., 2019]). Por exemplo, algoritmos baseados em árvores necessitam que sejam definidos valores para profundidade máxima, número máximo de nós-folha e critério para medir a qualidade de 
uma divisão. A otimização manual desses hiperparâmetros é uma tarefa repetitiva, tediosa e com sucesso puramente baseado no acaso ou experiência.

O número de modelos testados pela QuickAutoML é igual ao número de algoritmos multiplicado pelo número de combinações de hiperparâmetros possíveis. O modelo com o melhor desempenho é resultante da combinação de hiperparâmetros ótimos para determinado algoritmo. Para evitar problemas pós-treinamento, como overfitting (i.e., condição na qual o modelo se ajusta bem nos dados de treino, porém perde sua capacidade de generalização para dados novos), é utilizada uma abordagem chamada validação cruzada com $k$ dobras. Essa abordagem adiciona uma camada de treinamento extra ao particionar os dados $k$ vezes, de acordo com a necessidade.

Na ferramenta, utilizamos $k=5$ por ser um valor tipicamente utilizado e apresentar tempo menor de execução em comparação a outras técnicas de validação (e.g., LOOCV) [James et al., 2013]. Na prática, isso significa dividir o conjunto de dados em cinco subconjuntos e efetuar cinco rodadas de treino e validação. Em cada rodada, o modelo é treinado com quatro dessas dobras e validado com a dobra restante. Ao final, é agregada a acurácia média das avaliações. $\mathrm{O}$ modelo que possuir a maior acurácia média é escolhido.

A ferramenta foi desenvolvida de forma a permitir que, além da lista pré-definida de algoritmos candidatos a modelo, o usuário utilize implementações de outras bibliotecas ou mesmo seus próprios algoritmos, desde que estejam implementados de acordo com a estrutura que a ferramenta suporta.

\section{Avaliação}

Para avaliar o desempenho da QuickAutoML em termos de acurácia e tempo para execução, realizamos uma comparação com a ferramenta TPOT. A metodologia do experimento é descrita a seguir.

\subsection{Metodologia}

Utilizamos os seis datasets (new_thyroid, hypothyroid, dermatology, hayes_roth, dis e penguins) criados e disponibilizados ${ }^{1}$ para fins de comparação entre modelos de aprendizado de máquina [Olson et al., 2017]. O tempo de execução (em segundos) e a acurácia de ambas as ferramentas foram medidos para cada um dos conjuntos de dados. Os resultados numéricos representam a média de cinco execuções para cada dataset. Além disso, para fins de reprodutibilidade do experimento, foi utilizado random_st at $e=777$ e $\mathrm{CV}=5$. A Tabela 1 apresenta os detalhes sobre cada um dos conjuntos de dados.

Tabela 1. Datasets utilizados para avaliação da ferramenta

\begin{tabular}{|c|c|c|c|c|}
\hline Nome & $\begin{array}{c}\text { Número de } \\
\text { amostras }\end{array}$ & $\begin{array}{c}\text { Número de } \\
\text { features }\end{array}$ & $\begin{array}{c}\text { Número de } \\
\text { classes }\end{array}$ & Balanceamento \\
\hline new_thyroid & 215 & 5 & 3 & 0.299080 \\
\hline hypothyroid & 3163 & 25 & 2 & 0.818158 \\
\hline dermatology & 366 & 34 & 6 & 0.041458 \\
\hline hayes_roth & 160 & 4 & 3 & 0.043867 \\
\hline dis & 3772 & 29 & 2 & 0.939439 \\
\hline penguins & 344 & 7 & 3 & 0.046376 \\
\hline
\end{tabular}

${ }^{1}$ https://github.com/EpistasisLab/pmlb/tree/master/datasets 
Pelo fato de TPOT otimizar as etapas de pré-processamento de dados, seleção de características e algoritmos de aprendizado de máquina através de programação genética, a execução pode levar horas ou dias para ser concluída; portanto, para fins de ajuste de escala na comparação do tempo de execução, foi utilizada uma configuração de classificador do TPOT descrita na documentação ${ }^{2}$, com generations $=5$ e population_size=20.

A configuração do ambiente utilizado para o experimento é: processador 11 th Gen Intel(R) Core(TM) i7-1185G7 @ 3.00GHz, 32GB de memória RAM, Python 3.8.10, PyCharm IDE 212.5080.64, JRE 11.0.11 e Ubuntu 20.04.2 LTS, assim como as bibliotecas NumPy 1.19.5, scikit-learn 0.24.2 e pandas 1.1.5.

\subsection{Resultados}

Pelos resultados sumarizados na Tabela 2, podemos observar que a acurácia obtida pela QuickAutoML é equiparada à obtida pela TPOT em cinco dos seis datasets, sendo a maior diferença de $6.25 \%$, em "hayes_roth". Entretanto, vale destacar o tempo necessário para execução da TPOT que, no pior caso, apresentou uma diferença de 201.62 segundos em relação a QuickAutoML; este tempo pode ainda crescer exponencialmente, dependendo do tamanho do conjunto de dados utilizado. O principal gargalo da TPOT é originado pela otimização dos pipelines, processo este que a QuickAutoML não implementa, por isso, o tempo de execução nesta é consideravelmente inferior. A configuração do classificador da TPOT, descrita na Subseção 3.1, define que o mesmo irá buscar o melhor pipeline entre 100. Se utilizarmos o valor definido por padrão (generations=100 e population_size=100), o número de pipelines salta para 10,000. Com isso, mesmo em datasets consideravelmente pequenos, o tempo de execução na TPOT pode ser significativamente maior.

Tabela 2. Resultados obtidos na comparação entre QuickAutoML e TPOT

\begin{tabular}{|l|c|c|c|c|}
\hline \multicolumn{1}{|c|}{ dataset } & $\begin{array}{c}\text { Tempo } \\
\text { (QuickAutoML) }\end{array}$ & $\begin{array}{c}\text { Acurácia } \\
\text { (QuickAutoML) }\end{array}$ & $\begin{array}{c}\text { Tempo } \\
\text { (TPOT) }\end{array}$ & $\begin{array}{c}\text { Acurácia } \\
\text { (TPOT) }\end{array}$ \\
\hline new_thyroid & 3.6433 & 0.9535 & 29.8389 & 0.9535 \\
\hline hypothyroid & 7.1176 & 0.9858 & 158.2114 & 0.9905 \\
\hline dermatology & 4.2010 & 0.9730 & 54.8852 & 0.9595 \\
\hline hayes_roth & 3.9715 & 0.6875 & 29.4409 & 0.75 \\
\hline dis & 7.6694 & 0.9881 & 209.2988 & 0.9907 \\
\hline penguins & 3.3933 & 0.9551 & 40.5660 & 0.9701 \\
\hline
\end{tabular}

\section{Trabalhos relacionados}

Na Tabela 3 resumimos informações sobre ferramentas para automatização do processo de treinamento, avaliação e manutenção de modelos de aprendizado de máquina. As ferramentas foram agrupadas em três categorias, de acordo com a finalidade de sua utilização: AutoML, gerenciamento de modelos e avaliação de modelos.

Trabalhos recentes (e.g., auto-sklearn [Feurer et al., 2015] e TPOT [Olson and Moore, 2016]) propõem soluções para automatizar processos de aprendizado de máquina, como seleção de características, processamento de dados, teste e otimização de modelos, implementando um conceito conhecido como AutoML. A

${ }^{2}$ http://epistasislab.github.io/tpot/api/\#classification 
Tabela 3. Principais características de trabalhos relacionados

\begin{tabular}{|c|c|c|c|c|c|c|c|}
\hline Nome & Open Source & Ano & $\begin{array}{c}\text { Dados estruturados } \\
\text { (e.g. arquivos .csv) }\end{array}$ & $\begin{array}{c}\text { Dados não estruturados } \\
\text { (e.g. imagens, texto) }\end{array}$ & Supervisionado & $\begin{array}{c}\text { Não } \\
\text { supervisionado }\end{array}$ & Categoria \\
\hline QuickAutoML & Não & 2021 & Sim & Não & Sim & Não & AutoML \\
\hline Auto-sklearn & Sim & 2015 & Sim & Não & Sim & Não \\
\hline TPOT & Sim & 2016 & Sim & Não & Sim & Não & Gim \\
\hline Runway & Não & 2018 & Sim & Não & Sim & Não \\
\hline TFX & Sim & 2017 & Sim & Sim & Sim & Não \\
\hline Gestalt & Não & 2010 & Sim & Sim & Sim & Não \\
\hline ModelDB & Sim & 2016 & Sim & Sim & Sim & Não \\
\hline ModelHub & Sim & 2016 & Sim & Sim & Sim & Não \\
\hline Foolbox & Sim & 2017 & Sim & Sim & Sim & Não \\
\hline DroidAPIMiner & Sim & 2017 & Não & Sim & Sim & Não \\
\hline ModelTracker & Não & 2015 & Sim & Sim & Sim & Não & \\
\hline CrossCheck & Sim & 2020 & Sim & Não & & \\
\hline
\end{tabular}

utilização de AutoML permite que usuários desenvolvam soluções baseados em aprendizado de máquina sem conhecimentos técnicos aprofundados. Diferentemente destas, o objetivo da QuickAutoML é fornecer bons resultados com um tempo de execução significativamente menor através da redução do número de etapas de pré-processamento de dados.

Já ferramentas como ModelTracker [Amershi et al., 2015], Crosscheck [Arendt et al., 2020] e Gestalt [Patel et al., 2010] focam na avaliação e validação dos resultados através de interfaces gráficas, objetivando tornar a avaliação dos modelos mais intuitiva e eficiente. Similarmente, atráves da QuickAutoML o usuário pode gerar relatórios de desempenho dos modelos treinados em formato de gráficos ou planilhas, o que facilita a análise dos resultados.

Outras ferramentas, como Runway [Tsay et al., 2018], ModelHub [Miao et al., 2016] e ModelDB [Vartak et al., 2016], atuam no gerenciamento de modelos após as etapas de treinamento, facilitando a rastreabilidade e comparação entre os modelos. Isto é importante considerando o cenário dinâmico onde os modelos de aprendizado de máquina estão inseridos, podendo se tornar ineficientes à medida que os dados ou requisitos mudam [Paleyes et al., 2020]. Futuramente, a QuickAutoML fornecerá suporte para o gerenciamento dos modelos criados, isto é, oferecerá um maior suporte à manutenibilidade e a rastreabilidade destes ao decorrer do tempo.

\section{Considerações Finais}

Neste trabalho, apresentamos a QuickAutoML, uma ferramenta para automatização do processo de criação e treinamento de modelos de aprendizado de máquina. Os resultados indicam que a ferramenta consegue encontrar um modelo muito bom para diversos tipos de problemas sem necessidade de ajustes manuais e com um tempo de execução consideravelmente inferior quando comparado com ferramentas como a TPOT.

Para dar continuidade ao desenvolvimento da ferramenta, podemos elencar os seguintes trabalhos futuros: (a) fornecer suporte a outras abordagens de aprendizado de máquina, como métodos de regressão e aprendizado profundo (deep learning); (b) implementar etapas de limpeza de dados, criação, transformação e seleção de características ( $f e$ atures); e (c) fornecer as funcionalidades através de uma interface gráfica para o usuário.

\section{Agradecimentos}

Esta pesquisa foi financiada, conforme previsto nos Arts. 21 e 22 do decreto no. 10.521/2020, nos termos da Lei Federal no. 8.387/1991, através do convênio no. 
003/2021, firmado entre ICOMP/UFAM, Flextronics da Amazônia Ltda e Motorola Mobility Comércio de Produtos Eletrônicos Ltda.

\section{Referências}

Amershi, S., Chickering, M., Drucker, S. M., Lee, B., Simard, P., and Suh, J. (2015). Modeltracker: Redesigning performance analysis tools for machine learning. In Proceedings of the 33rd Annual ACM Conference on Human Factors in Computing Systems, pages 337-346.

Arendt, D., Huang, Z., Shrestha, P., Ayton, E., Glenski, M., and Volkova, S. (2020). Crosscheck: Rapid, reproducible, and interpretable model evaluation. arXiv preprint arXiv:2004.07993.

Botchkarev, A. (2018). Performance metrics (error measures) in machine learning regression, forecasting and prognostics: Properties and typology. arXiv preprint arXiv:1809.03006.

Caruana, R. and Niculescu-Mizil, A. (2006). An empirical comparison of supervised learning algorithms. In Proceedings of the 23rd international conference on Machine learning, pages 161-168.

Feurer, M., Klein, A., Eggensperger, K., Springenberg, J., Blum, M., and Hutter, F. (2015). Efficient and robust automated machine learning. In Advances in Neural Information Processing Systems 28, pages 2962-2970. Curran Associates, Inc.

James, G., Witten, D., Hastie, T., and Tibshirani, R. (2013). An introduction to statistical learning, volume 112. Springer.

Miao, H., Li, A., Davis, L. S., and Deshpande, A. (2016). Modelhub: Towards unified data and lifecycle management for deep learning. arXiv preprint arXiv:1611.06224.

Olson, R. S., La Cava, W., Orzechowski, P., Urbanowicz, R. J., and Moore, J. H. (2017). Pmlb: a large benchmark suite for machine learning evaluation and comparison. BioData mining, 10(1):1-13.

Olson, R. S. and Moore, J. H. (2016). Tpot: A tree-based pipeline optimization tool for automating machine learning. In Workshop on automatic machine learning, pages 66-74. PMLR.

Paleyes, A., Urma, R.-G., and Lawrence, N. D. (2020). Challenges in deploying machine learning: a survey of case studies. arXiv preprint arXiv:2011.09926.

Patel, K., Bancroft, N., Drucker, S. M., Fogarty, J., Ko, A. J., and Landay, J. (2010). Gestalt: integrated support for implementation and analysis in machine learning. In Proceedings of the 23nd annual ACM symposium on User interface software and technology, pages 37-46.

Pedregosa, F., Varoquaux, G., Gramfort, A., Michel, V., Thirion, B., Grisel, O., Blondel, M., Prettenhofer, P., Weiss, R., Dubourg, V., Vanderplas, J., Passos, A., Cournapeau, D., Brucher, M., Perrot, M., and Duchesnay, E. (2011). Scikit-learn: Machine learning in Python. Journal of Machine Learning Research, 12:2825-2830.

Tsay, J., Mummert, T., Bobroff, N., Braz, A., Westerink, P., and Hirzel, M. (2018). Runway: machine learning model experiment management tool. In Conference on Systems and Machine Learning (SysML).

Vartak, M., Subramanyam, H., Lee, W.-E., Viswanathan, S., Husnoo, S., Madden, S., and Zaharia, M. (2016). Modeldb: a system for machine learning model management. In Proceedings of the Workshop on Human-In-the-Loop Data Analytics, pages 1-3.

Wu, J., Chen, X.-Y., Zhang, H., Xiong, L.-D., Lei, H., and Deng, S.-H. (2019). Hyperparameter optimization for machine learning models based on bayesian optimization. Journal of Electronic Science and Technology, 17(1):26-40.

Yao, X. and Liu, Y. (2014). Machine learning. In Search Methodologies, pages 477-517. Springer. 